\title{
Gradient estimates and Liouville-type theorems for a weighted nonlinear elliptic equation
}

\author{
Bingqing $\mathrm{Ma}^{1,2^{*}}$ and Yongli Dong ${ }^{2}$
}

\author{
"Correspondence: \\ bqma@henannu.edu.cn \\ ${ }^{1}$ College of Physics and Materials \\ Science, Henan Normal University, \\ Xinxiang, P.R. China \\ ${ }^{2}$ Department of Mathematics, \\ Henan Normal University, Xinxiang, \\ P.R. China
}

\begin{abstract}
We consider gradient estimates for positive solutions to the following nonlinear elliptic equation on a smooth metric measure space $\left(M, g, e^{-f} d v\right)$ :

$$
\Delta_{f} u+a u \log u+b u=0
$$

where $a, b$ are two real constants. When the $\infty$-Bakry-Émery Ricci curvature is bounded from below, we obtain a global gradient estimate which is not dependent on $|\nabla f|$. In particular, we find that any bounded positive solution of the above equation must be constant under some suitable assumptions.
\end{abstract}

MSC: Primary 58J35; secondary 35B45

Keywords: Gradient estimate; Nonlinear elliptic equation; Liouville-type theorem

\section{Introduction}

Let $(M, g)$ be an $n$-dimensional complete Riemannian manifold and $f$ be a smooth function defined on $M$. Then the triple $\left(M, g, e^{-f} d v\right)$ is called a smooth metric measure space, where $d v$ denotes the volume element of the metric $g$ and $e^{-f} d v$ is called the weighted measure. On the smooth metric measure space $\left(M, g, e^{-f} d v\right)$, the $m$-Bakry-Émery Ricci curvature (see [1-3]) is defined by

$$
\operatorname{Ric}_{f}^{m}=\operatorname{Ric}+\nabla^{2} f-\frac{1}{m-n} d f \otimes d f
$$

where $m \geq n$ is a constant, and $m=n$ if and only if $f$ is a constant. We define

$$
\operatorname{Ric}_{f}=\operatorname{Ric}+\nabla^{2} f
$$

Then $\operatorname{Ric}_{f}$ can be seen as the $\infty$-dimensional Bakry-Émery Ricci curvature. However, there are many differences between the $m$-Bakry-Émery Ricci curvature and the $\infty$ Bakry-Émery Ricci curvature. For example, there exist complete noncompact Riemannian manifolds which satisfy $\operatorname{Ric}_{f}=\lambda g$ for some positive constant $\lambda$ (which is called the shrinking gradient Ricci soliton), but not for $\operatorname{Ric}_{f}^{m}=\lambda g$. We recall that the $f$-Laplacian $\Delta_{f}$

(c) The Author(s) 2018. This article is distributed under the terms of the Creative Commons Attribution 4.0 International License (http://creativecommons.org/licenses/by/4.0/), which permits unrestricted use, distribution, and reproduction in any medium, provided you give appropriate credit to the original author(s) and the source, provide a link to the Creative Commons license, and indicate if changes were made. 
on $\left(M, g, e^{-f} d v\right)$ is defined by

$$
\Delta_{f}=\Delta-\nabla f \nabla .
$$

Since we have the Bochner formula with respect to $f$-Laplacian:

$$
\frac{1}{2} \Delta_{f}|\nabla u|^{2} \geq \frac{1}{m}\left(\Delta_{f} u\right)^{2}+\nabla u \nabla\left(\Delta_{f} u\right)+\operatorname{Ric}_{f}^{m}(\nabla u, \nabla u)
$$

which is similar to the Bochner formula associated with the Laplacian, many results with respect to the Laplacian have been generalized to those of the $f$-Laplacian under the $m$ dimensional Bakry-Émery Ricci curvature. For example, see [4-7] and the references therein. But for elliptic gradient estimates for $f$-Laplacian under the $\infty$-Bakry-Émery Ricci curvature, in order to using the weighted comparison theorem, the assumption $|\nabla f| \leq \theta$ is forced commonly.

In this paper, under the assumption that the $\infty$-Bakry-Émery Ricci curvature is bounded from below, we consider the following nonlinear elliptic equation:

$$
\Delta_{f} u+a u \log u+b u=0
$$

where $a, b$ are two real constants. Inspired by the ideas of Brighton in [8], we can obtain global gradient estimates for positive solutions to (1.3) without any restriction on $|\nabla f|$.

Theorem 1.1 Let $\left(M, g, e^{-f} d v\right)$ be an $n$-dimensional complete smooth metric measure space with $\operatorname{Ric}_{f}\left(B_{p}(2 R)\right) \geq-(n-1) K$, where $K \geq 0$ is a constant. Suppose that $u$ is a positive solution to (1.3) with $u \leq A$ on $B_{p}(2 R)$. Then on $B_{p}(R)$ with $R>1$, the following inequality holds:

$$
|\nabla u|^{2} \leq C A^{2}\left[\max \left\{\frac{4}{5} b+a\left(1+\frac{4}{5} L\right), 0\right\}+K+\frac{|\beta|+1}{R}\right]
$$

where $C$ is a positive constant which depends on the dimension $n, \beta=\max _{\{x \mid d(x, p)=1\}} \Delta_{f} r(x)$ and

$$
L= \begin{cases}\sup _{B_{p}(2 R)}(\log u), & \text { if } a \geq 0, \\ \inf _{B_{p}(2 R)}(\log u), & \text { if } a<0 .\end{cases}
$$

Letting $R \rightarrow \infty$ in (1.4), we obtain the following global estimates on complete noncompact Riemannian manifolds:

Corollary 1.2 Let $\left(M, g, e^{-f} d v\right)$ be an $n$-dimensional complete smooth metric measure space with $\operatorname{Ric}_{f} \geq-(n-1) K$, where $K \geq 0$ is a constant. If $u$ is a positive solution to (1.3) with $u \leq A$, then we have

$$
|\nabla u|^{2} \leq C A^{2}\left[\max \left\{\frac{4}{5} b+a\left(1+\frac{4}{5} L\right), 0\right\}+K\right],
$$


where

$$
L= \begin{cases}\sup _{M}(\log u), & \text { if } a \geq 0, \\ \inf _{M}(\log u), & \text { if } a<0 .\end{cases}
$$

Using the ideas of the proof of Theorem 1.1, by choosing $\tilde{h}=\log u$ a gap develops between the constants, and we also establish the following.

Theorem 1.3 Let $\left(M, g, e^{-f} d v\right)$ be an $n$-dimensional complete smooth metric measure space with $\operatorname{Ric}_{f}\left(B_{p}(2 R)\right) \geq-(n-1) K$, where $K \geq 0$ is a constant. Suppose that $u$ is a positive solution to (1.3) on $B_{p}(2 R)$ such that:

(1) either $\nabla f \nabla(\log u)-a \log u-b \leq \delta|\nabla(\log u)|^{2}$ for some $0 \leq \delta<\frac{1}{2}$;

(2) or $\nabla f \nabla(\log u)-a \log u-b \geq 2|\nabla(\log u)|^{2}$.

Then on $B_{p}(R)$ with $R>1$, the following inequality holds:

$$
|\nabla(\log u)|^{2} \leq \frac{C_{1}(n, \delta, \beta)}{R}+C_{2}(n, \delta) \max \{a+(n-1) K, 0\},
$$

where $\beta=\max _{\{x \mid d(x, p)=1\}} \Delta_{f} r(x)$.

Letting $R \rightarrow \infty$ in (1.8), we obtain the following global estimates on complete noncompact Riemannian manifolds:

Corollary 1.4 Let $\left(M, g, e^{-f} d v\right)$ be an $n$-dimensional complete smooth metric measure space with $\operatorname{Ric}_{f} \geq-(n-1) K$, where $K \geq 0$ is a constant. Let $u$ be a positive solution to (1.3). Then under the assumption of either (1) or (2) as in Theorem 1.3, we have

$$
|\nabla(\log u)|^{2} \leq C(n, \delta) \max \{a+(n-1) K, 0\}
$$

Clearly, if either $u \leq e^{-\left(\frac{5}{4}+\frac{b}{a}\right)}$ and $a>0$, or $u \geq e^{-\left(\frac{5}{4}+\frac{b}{a}\right)}$ and $a<0$, then we have $\frac{4}{5} b+$ $a\left(1+\frac{4}{5} L\right) \leq 0$. This gives the following result.

Corollary 1.5 Let $\left(M, g, e^{-f} d v\right)$ be an n-dimensional complete smooth metric measure space with $\operatorname{Ric}_{f} \geq 0$.

(1) There exists no bounded positive solution to (1.3) with $a>0$ and $u \leq e^{-\left(\frac{5}{4}+\frac{b}{a}\right) \text {; }}$

(2) if $a<0$ and $u \geq e^{-\left(\frac{5}{4}+\frac{b}{a}\right)}$, then any bounded positive solution to (1.3) must be constant $u=e^{-\frac{b}{a}}$.

Remark 1.1 In particular, when $a=0$, Eq. (1.3) becomes

$$
\Delta_{f} u+b u=0
$$

and (1.6) becomes

$$
|\nabla u|^{2} \leq C A^{2}\left[\max \left\{\frac{4}{5} b, 0\right\}+K\right] .
$$


In this case, on a complete smooth metric measure space $\left(M, g, e^{-f} d v\right)$ with $\operatorname{Ric}_{f} \geq 0$, there exists no bounded positive solution to (1.10) with $b<0$. On the other hand, if $a=b=0$, our Theorem 1.1 becomes Theorem 1 of Brighton in [8].

Remark 1.2 It is easy to see from Corollary 1.4 that if $u$ is a positive solution to (1.3) with $a \leq-(n-1) K$ satisfying either (1) or (2) in Theorem 1.3 , then $u=e^{-\frac{b}{a}}$ is a constant. In particular, if $a=b=0$, then our Theorem 1.3 becomes Theorem 3 of Brighton in [8].

Remark 1.3 Some related results for gradient estimates of positive solutions to

$$
\Delta_{f} u+a u \log u=0
$$

can be found in [9-11]. Moreover, Qian in [10] used a different method to derive similar estimates to (1.12) with constant $f$. On the other hand, if we assume $\operatorname{Ric}_{f} \geq-(n-1) K$ and $|\nabla f| \leq \theta$, then from (1.1), we obtain

$$
\begin{aligned}
\operatorname{Ric}_{f}^{m} & =\operatorname{Ric}_{f}-\frac{1}{m-n} d f \otimes d f \\
& \geq-(n-1)\left(K+\frac{\theta^{2}}{(m-n)(n-1)}\right):=-(n-1) \tilde{K}
\end{aligned}
$$

Hence, Theorem 1.5 in [11] follows from Theorem 1.1 of [11] immediately. However, our estimates in this paper are not dependent on $|\nabla f|$.

\section{Proof of results}

We firstly give the following lemma which plays an important role in the proof of main results.

Lemma 2.1 Let $u$ be a positive solution to (1.3) with $u \leq A$ and $\operatorname{Ric}_{f} \geq-(n-1) K$ for some positive constant $K$. Denote $\tilde{u}=u / A$ and $h=\tilde{u}^{\epsilon}$ for $\epsilon \in(0,1)$. If there exists one positive constant $\delta$ satisfying

$$
\frac{1}{n}+\frac{2(\epsilon-1)}{n \epsilon \delta} \geq 0
$$

then we have

$$
\begin{aligned}
\frac{1}{2} \Delta_{f}|\nabla h|^{2} \geq & \left(\frac{(\epsilon-1)^{2}}{n \epsilon^{2}}-\frac{\epsilon-1}{\epsilon}+\frac{2 \delta(\epsilon-1)}{n \epsilon}\right) \frac{|\nabla h|^{4}}{h^{2}}+\frac{\epsilon-1}{\epsilon} \frac{\nabla h}{h} \nabla\left(|\nabla h|^{2}\right) \\
& -[a+\tilde{b} \epsilon+(n-1) K+a \tilde{L}]|\nabla h|^{2},
\end{aligned}
$$

where

$$
\tilde{L}= \begin{cases}\sup _{M}(\log h), & \text { if } a \geq 0, \\ \inf _{M}(\log h), & \text { if } a<0 .\end{cases}
$$

Proof Under the scaling $u \rightarrow \tilde{u}=u / A$, it follows from (1.3) that $\tilde{u}$ satisfies

$$
\Delta_{f} \tilde{u}+a \tilde{u} \log \tilde{u}+\tilde{b} \tilde{u}=0,
$$


where the constant $\tilde{b}$ is given by $\tilde{b}=b+a \log A$. Let $h=\tilde{u}^{\epsilon}$, where $\epsilon \in(0,1)$ is a constant to be determined. Then we have

$$
\log h=\epsilon \log \tilde{u} .
$$

Since $0<\tilde{u} \leq 1$, we have $\log h \leq 0$ and

$$
\begin{aligned}
\Delta_{f} h & =\Delta_{f}\left(\tilde{u}^{\epsilon}\right)=\epsilon(\epsilon-1) \tilde{u}^{\epsilon-2}|\nabla \tilde{u}|^{2}+\epsilon \tilde{u}^{\epsilon-1} \Delta_{f} \tilde{u} \\
& =\epsilon(\epsilon-1) \tilde{u} \tilde{u}^{\epsilon-2}|\nabla \tilde{u}|^{2}-a \epsilon \tilde{u} \log \tilde{u}-\tilde{b} \epsilon \tilde{u}^{\epsilon} \\
& =\frac{\epsilon-1}{\epsilon} \frac{|\nabla h|^{2}}{h}-a h \log h-\tilde{b} \epsilon h,
\end{aligned}
$$

which implies

$$
\begin{aligned}
\nabla h \nabla \Delta_{f} h= & \nabla h \nabla\left(\frac{\epsilon-1}{\epsilon} \frac{|\nabla h|^{2}}{h}-a h \log h-\tilde{b} \epsilon h\right) \\
= & \frac{\epsilon-1}{\epsilon} \nabla h \nabla \frac{|\nabla h|^{2}}{h}-a \nabla h \nabla(h \log h)-\tilde{b} \epsilon|\nabla h|^{2} \\
= & \frac{\epsilon-1}{\epsilon} \frac{\nabla h}{h} \nabla\left(|\nabla h|^{2}\right)-\frac{\epsilon-1}{\epsilon} \frac{|\nabla h|^{4}}{h^{2}} \\
& -a h \log h \frac{|\nabla h|^{2}}{h}-(a+\tilde{b} \epsilon)|\nabla h|^{2} .
\end{aligned}
$$

Thus, under the assumption $\operatorname{Ric}_{f} \geq-(n-1) K$, one has

$$
\begin{aligned}
\frac{1}{2} \Delta_{f}|\nabla h|^{2}= & \left|\nabla^{2} h\right|^{2}+\nabla h \nabla \Delta_{f} h+\operatorname{Ric}_{f}(\nabla h, \nabla h) \\
\geq & \frac{1}{n}(\Delta h)^{2}+\nabla h \nabla \Delta_{f} h-(n-1) K|\nabla h|^{2} \\
= & \frac{1}{n}\left(\frac{\epsilon-1}{\epsilon} \frac{|\nabla h|^{2}}{h}+\nabla f \nabla h-a h \log h-\tilde{b} \epsilon h\right)^{2}+\frac{\epsilon-1}{\epsilon} \frac{\nabla h}{h} \nabla\left(|\nabla h|^{2}\right) \\
& -\frac{\epsilon-1}{\epsilon} \frac{|\nabla h|^{4}}{h^{2}}-(a h \log h) \frac{|\nabla h|^{2}}{h}-[a+\tilde{b} \epsilon+(n-1) K]|\nabla h|^{2} \\
= & \left(\frac{(\epsilon-1)^{2}}{n \epsilon^{2}}-\frac{\epsilon-1}{\epsilon}\right) \frac{|\nabla h|^{4}}{h^{2}}+\frac{2(\epsilon-1)}{n \epsilon} \frac{|\nabla h|^{2}}{h}(\nabla f \nabla h-a h \log h-\tilde{b} \epsilon h) \\
& +\frac{1}{n}(\nabla f \nabla h-a h \log h-\tilde{b} \epsilon h)^{2}+\frac{\epsilon-1}{\epsilon} \frac{\nabla h}{h} \nabla\left(|\nabla h|^{2}\right) \\
& -[a+\tilde{b} \epsilon+(n-1) K+a \log h]|\nabla h|^{2} .
\end{aligned}
$$

For any fixed point $p$, if there exists a positive constant $\delta$ such that $\nabla f \nabla h-a h \log h-\tilde{b} \in h \leq$ $\delta \frac{|\nabla h|^{2}}{h}$, then from (2.8), we can deduce

$$
\begin{aligned}
\frac{1}{2} \Delta_{f}|\nabla h|^{2} \geq & \left(\frac{(\epsilon-1)^{2}}{n \epsilon^{2}}-\frac{\epsilon-1}{\epsilon}\right) \frac{|\nabla h|^{4}}{h^{2}}+\frac{2(\epsilon-1)}{n \epsilon} \frac{|\nabla h|^{2}}{h}\left(\delta \frac{|\nabla h|^{2}}{h}\right) \\
& +\frac{1}{n}(\nabla f \nabla h-a h \log h-\tilde{b} \epsilon h)^{2}+\frac{\epsilon-1}{\epsilon} \frac{\nabla h}{h} \nabla\left(|\nabla h|^{2}\right)
\end{aligned}
$$




$$
\begin{aligned}
& -[a+\tilde{b} \epsilon+(n-1) K+a \log h]|\nabla h|^{2} \\
\geq & \left(\frac{(\epsilon-1)^{2}}{n \epsilon^{2}}-\frac{\epsilon-1}{\epsilon}+\frac{2 \delta(\epsilon-1)}{n \epsilon}\right) \frac{|\nabla h|^{4}}{h^{2}}+\frac{\epsilon-1}{\epsilon} \frac{\nabla h}{h} \nabla\left(|\nabla h|^{2}\right) \\
& -[a+\tilde{b} \epsilon+(n-1) K+a \tilde{L}]|\nabla h|^{2} .
\end{aligned}
$$

On the contrary, if $\nabla f \nabla h-a h \log h-\tilde{b} \in h \geq \delta \frac{|\nabla h|^{2}}{h}$ at the point $p$, then from (2.8), we can deduce

$$
\begin{aligned}
\frac{1}{2} \Delta_{f}|\nabla h|^{2} \geq & \left(\frac{(\epsilon-1)^{2}}{n \epsilon^{2}}-\frac{\epsilon-1}{\epsilon}\right) \frac{|\nabla h|^{4}}{h^{2}}+\frac{2(\epsilon-1)}{n \epsilon \delta}(\nabla f \nabla h-a h \log h-\tilde{b} \epsilon h)^{2} \\
& +\frac{1}{n}(\nabla f \nabla h-a h \log h-\tilde{b} \epsilon h)^{2}+\frac{\epsilon-1}{\epsilon} \frac{\nabla h}{h} \nabla\left(|\nabla h|^{2}\right) \\
& -[a+\tilde{b} \epsilon+(n-1) K+a \log h]|\nabla h|^{2} \\
= & \left(\frac{(\epsilon-1)^{2}}{n \epsilon^{2}}-\frac{\epsilon-1}{\epsilon}\right) \frac{|\nabla h|^{4}}{h^{2}}+\left(\frac{1}{n}+\frac{2(\epsilon-1)}{n \epsilon \delta}\right)(\nabla f \nabla h-a h \log h-\tilde{b} \epsilon h)^{2} \\
& +\frac{\epsilon-1}{\epsilon} \frac{\nabla h}{h} \nabla\left(|\nabla h|^{2}\right)-[a+\tilde{b} \epsilon+(n-1) K+a \log h]|\nabla h|^{2} \\
\geq & \left.\frac{(\epsilon-1)^{2}}{n \epsilon^{2}}-\frac{\epsilon-1}{\epsilon}+\left(\frac{1}{n}+\frac{2(\epsilon-1)}{n \epsilon \delta}\right) \delta^{2}\right] \frac{|\nabla h|^{4}}{h^{2}}+\frac{\epsilon-1}{\epsilon} \frac{\nabla h}{h} \nabla\left(|\nabla h|^{2}\right) \\
& -[a+\tilde{b} \epsilon+(n-1) K+a \log h]|\nabla h|^{2} \\
\geq & \left(\frac{(\epsilon-1)^{2}}{n \epsilon^{2}}-\frac{\epsilon-1}{\epsilon}+\frac{2 \delta(\epsilon-1)}{n \epsilon}\right) \frac{|\nabla h|^{4}}{h^{2}}+\frac{\epsilon-1}{\epsilon} \frac{\nabla h}{h} \nabla\left(|\nabla h|^{2}\right) \\
& -[a+\tilde{b} \epsilon+(n-1) K+a \tilde{L}]|\nabla h|^{2}
\end{aligned}
$$

as long as (2.1) holds.

Therefore, in these two cases the estimate (2.2) holds, which finishes the proof of the Lemma 2.1.

\subsection{Proof of Theorem 1.1}

In order to obtain the upper bound of $|\nabla h|$ by using the maximum principle for (2.2), we need to choose $\epsilon, \delta$ such that the coefficient of $\frac{|\nabla h|^{4}}{h^{2}}$ in (2.2) is positive. That is, we need

$$
\frac{(\epsilon-1)^{2}}{n \epsilon^{2}}-\frac{\epsilon-1}{\epsilon}+\frac{2 \delta(\epsilon-1)}{n \epsilon}>0 .
$$

In particular, by choosing $\epsilon=\frac{4}{5}$ and letting $\delta \rightarrow \frac{1}{2}$, we find that the inequality (2.1) holds and (2.2) becomes

$$
\begin{aligned}
\frac{1}{2} \Delta_{f}|\nabla h|^{2} \geq & \frac{4 n-3}{16 n} \frac{|\nabla h|^{4}}{h^{2}}-\frac{1}{4} \frac{\nabla h}{h} \nabla\left(|\nabla h|^{2}\right) \\
& -[a+\tilde{b} \epsilon+(n-1) K+a \tilde{L}]|\nabla h|^{2} .
\end{aligned}
$$


As in [8], we define a cut-off function $\psi \in C^{2}([0,+\infty))$ by

$$
\psi(t)= \begin{cases}1, & t \in[0, R] \\ 0, & t \in[2 R,+\infty]\end{cases}
$$

satisfying $\psi(t) \in[0,1]$ and

$$
-\frac{C}{R} \leq \frac{\psi^{\prime}(t)}{\sqrt{\psi}} \leq 0, \quad\left|\psi^{\prime \prime}(t)\right| \leq \frac{C}{R^{2}}
$$

where $C$ is a positive constant. Let

$$
\phi=\psi(d(x, p))
$$

Using Eq. (2.19) in [8] (see Eq. (4.5) in [5] or [12, Theorem 3.1]), we obtain

$$
\Delta_{f} \phi \geq-\frac{C \beta}{R}-\frac{C(n-1) K(2 R-1)}{R}-\frac{C}{R^{2}}
$$

and

$$
\frac{|\nabla \phi|^{2}}{\phi} \leq \frac{C}{R^{2}}
$$

Denote by $B_{p}(R)$ the geodesic ball centered at $p$ with radius $R$. Let $G=\phi|\nabla h|^{2}$. Assume $G$ achieves its maximum at the point $x_{0} \in B_{p}(2 R)$ and assume $G\left(x_{0}\right)>0$ (otherwise the proof is trivial). Then, at the point $x_{0}$,

$$
\Delta_{f} G \leq 0, \quad \nabla\left(|\nabla h|^{2}\right)=-\frac{|\nabla h|^{2}}{\phi} \nabla \phi
$$

and

$$
\begin{aligned}
0 \geq & \Delta_{f} G \\
= & \phi \Delta_{f}\left(|\nabla h|^{2}\right)+|\nabla h|^{2} \Delta_{f} \phi+2 \nabla \phi \nabla|\nabla h|^{2} \\
= & \phi \Delta_{f}\left(|\nabla h|^{2}\right)+\frac{\Delta_{f} \phi}{\phi} G-2 \frac{|\nabla \phi|^{2}}{\phi^{2}} G \\
\geq & \frac{\Delta_{f} \phi}{\phi} G-2 \frac{|\nabla \phi|^{2}}{\phi^{2}} G+2 \phi\left[\frac{4 n-3}{16 n} \frac{|\nabla h|^{4}}{h^{2}}-\frac{1}{4} \frac{\nabla h}{h} \nabla\left(|\nabla h|^{2}\right)\right. \\
& \left.-[a+\tilde{b} \epsilon+(n-1) K+a \tilde{L}]|\nabla h|^{2}\right] \\
= & \frac{\Delta_{f} \phi}{\phi} G-2 \frac{|\nabla \phi|^{2}}{\phi^{2}} G+\frac{4 n-3}{8 n} \frac{G^{2}}{\phi h^{2}}+\frac{G}{2 \phi} \nabla \phi \frac{\nabla h}{h} \\
& -2[a+\tilde{b} \epsilon+(n-1) K+a \tilde{L}] G,
\end{aligned}
$$


where in the second inequality, we used (2.12). Multiplying both sides of (2.17) by $\frac{\phi}{G}$, we obtain

$$
\begin{aligned}
\frac{4 n-3}{8 n} \frac{G}{h^{2}} \leq & -\frac{1}{2} \nabla \phi \frac{\nabla h}{h}+2[a+\tilde{b} \epsilon+(n-1) K+a \tilde{L}] \phi \\
& -\Delta_{f} \phi+2 \frac{|\nabla \phi|^{2}}{\phi} .
\end{aligned}
$$

Substituting the Cauchy inequality

$$
\begin{aligned}
-\frac{1}{2} \nabla \phi \frac{\nabla h}{h} & \leq \frac{1}{2}|\nabla \phi| \frac{|\nabla h|}{h} \\
& \leq \frac{n}{4 n-3} \frac{|\nabla \phi|^{2}}{\phi}+\frac{4 n-3}{16 n} \phi \frac{|\nabla h|^{2}}{h^{2}} \\
& =\frac{n}{4 n-3} \frac{|\nabla \phi|^{2}}{\phi}+\frac{4 n-3}{16 n} \frac{G}{h^{2}}
\end{aligned}
$$

into (2.18) gives

$$
\begin{aligned}
\frac{4 n-3}{16 n} \frac{G}{h^{2}} & \leq 2[a+\tilde{b} \epsilon+(n-1) K+a \tilde{L}] \phi-\Delta_{f} \phi+\frac{9 n-6}{4 n-3} \frac{|\nabla \phi|^{2}}{\phi} \\
& \leq 2[a+\tilde{b} \epsilon+(n-1) K+a \tilde{L}]+\frac{C_{1}[(n-1) K(2 R-1)+\beta]}{R}+\frac{C_{2}}{R^{2}},
\end{aligned}
$$

where $C_{1}, C_{2}$ are two positive constants depending on $n$. Hence, on $B_{p}(R)$ with $R>1$, it follows from (2.19) that

$$
\begin{aligned}
\frac{4 n-3}{16 n} G(x) \leq & \frac{4 n-3}{16 n} G\left(x_{0}\right) \\
\leq & h^{2}\left(x_{0}\right)[2[a+\tilde{b} \epsilon+(n-1) K+a \tilde{L}] \\
& \left.+\frac{C_{1}[(n-1) K(2 R-1)+\beta]}{R}+\frac{C_{2}}{R^{2}}\right] .
\end{aligned}
$$

In particular, the estimate $(2.20)$ gives

$$
|\nabla u|^{2} \leq C A^{2}\left[\max \left\{\frac{4}{5} b+a\left(1+\frac{4}{5} L\right), 0\right\}+K+\frac{|\beta|+1}{R}\right],
$$

which finishes the proof of Theorem 1.1.

\subsection{Proof of Theorem 1.3}

We define $\tilde{h}=\log u$. Then we have

$$
\begin{aligned}
\Delta \tilde{h}-\nabla f \nabla \tilde{h} & =\Delta_{f} \tilde{h} \\
& =\frac{\Delta_{f} u}{u}-|\nabla(\log u)|^{2} \\
& =-|\nabla \tilde{h}|^{2}-a \tilde{h}-b,
\end{aligned}
$$


where, in the last equality of (2.22), we used Eq. (1.3). Using the Bochner formula with respect to the $f$-Laplacian, we have

$$
\begin{aligned}
\frac{1}{2} \Delta_{f}|\nabla \tilde{h}|^{2} & =\left|\nabla^{2} \tilde{h}\right|^{2}+\nabla \tilde{h} \nabla \Delta_{f} \tilde{h}+\operatorname{Ric}_{f}(\nabla \tilde{h}, \nabla \tilde{h}) \\
& \geq \frac{1}{n}(\Delta \tilde{h})^{2}+\nabla \tilde{h} \nabla \Delta_{f} \tilde{h}-(n-1) K|\nabla \tilde{h}|^{2} .
\end{aligned}
$$

Moreover, by virtue of (2.22), we have

$$
\begin{aligned}
(\Delta \tilde{h})^{2} & =\left(-|\nabla \tilde{h}|^{2}+\nabla f \nabla \tilde{h}-a \tilde{h}-b\right)^{2} \\
& =|\nabla \tilde{h}|^{4}-2|\nabla \tilde{h}|^{2}(\nabla f \nabla \tilde{h}-a \tilde{h}-b)+(\nabla f \nabla \tilde{h}-a \tilde{h}-b)^{2} .
\end{aligned}
$$

If the assumption (1) holds, then (2.24) yields

$$
\begin{aligned}
(\Delta \tilde{h})^{2} & \geq|\nabla \tilde{h}|^{4}-2 \delta|\nabla \tilde{h}|^{4}+(\nabla f \nabla \tilde{h}-a \tilde{h}-b)^{2} \\
& \geq(1-2 \delta)|\nabla \tilde{h}|^{4} .
\end{aligned}
$$

On the other hand, if the assumption (2) holds, then (2.24) shows

$$
\begin{aligned}
(\Delta \tilde{h})^{2} & \geq|\nabla \tilde{h}|^{4}-(\nabla f \nabla \tilde{h}-a \tilde{h}-b)^{2}+(\nabla f \nabla \tilde{h}-a \tilde{h}-b)^{2} \\
& =|\nabla \tilde{h}|^{4} \\
& \geq(1-2 \delta)|\nabla \tilde{h}|^{4} .
\end{aligned}
$$

Therefore, in these two cases, we have

$$
(\Delta \tilde{h})^{2} \geq(1-2 \delta)|\nabla \tilde{h}|^{4},
$$

and (2.23) gives

$$
\frac{1}{2} \Delta_{f}|\nabla \tilde{h}|^{2} \geq \frac{1-2 \delta}{n}|\nabla \tilde{h}|^{4}-\nabla \tilde{h} \nabla\left(|\nabla \tilde{h}|^{2}\right)-[a+(n-1) K]|\nabla \tilde{h}|^{2} .
$$

Following the proof of Theorem 1.1 line by line, we obtain on $B_{p}(R)$ with $R>1$,

$$
|\nabla \tilde{h}|^{2} \leq \frac{C_{1}(n, \delta, \beta)}{R}+C_{2}(n, \delta) \max \{a+(n-1) K, 0\}
$$

where $\delta$ is taken to zero in the second assumption.

We completed the proof of Theorem 1.3.

Acknowledgements

The authors want to thank the referee for helpful suggestions, which made the paper more readable. The research of the author is supported by NSFC No. 11401179.

The authors declare that they have no competing interests. 


\section{Publisher's Note}

Springer Nature remains neutral with regard to jurisdictional claims in published maps and institutional affiliations.

Received: 1 February 2018 Accepted: 27 April 2018 Published online: 10 May 2018

\section{References}

1. Bakry, D., Emery, M.: Diffusion hypercontractives. In: Sém. Prob. XIX. Lect. Notes in Math. 1123, pp. 177-206 (1985)

2. Bakry, D.: L'hypercontractivité et son utilisation en théorie des semigroupes. Lect. Notes Math. 1581, 1-114 (1994)

3. Li, X.-D.: Liouville theorems for symmetric diffusion operators on complete Riemannian manifolds. J. Math. Pures Appl. 84, 1361-1995 (2005)

4. Huang, G.Y., Li, Z.: Liouville type theorems of a nonlinear elliptic equation for the V-Laplacian. Anal. Math. Phys. 8, 123-134 (2018)

5. Wu, J.-Y.: Elliptic gradient estimates for a weighted heat equation and applications. Math. Z. 280, 451-468 (2015)

6. Huang, G.Y., Li, H.: Gradient estimates and entropy formulae of porous medium and fast diffusion equations for the Witten Laplacian. Pac. J. Math. 268, 47-78 (2014)

7. Li, X.-D.: Perelman's entropy formula for the Witten Laplacian on Riemannian manifolds via Bakry-Emery Ricci curvature. Math. Ann. 353, 403-437 (2012)

8. Brighton, K.: A Liouville-type theorem for smooth metric measure spaces. J. Geom. Anal. 23, 562-570 (2013)

9. Huang, G.Y., Ma, B.Q.: Gradient estimates and Liouville type theorems for a nonlinear elliptic equation. Arch. Math. (Basel) 105, 491-499 (2015)

10. Qian, B.: Yau's gradient estimates for a nonlinear elliptic equation. Arch. Math. (Basel) 108, 427-435 (2017)

11. Xiang, N., Chen, Y.: Liouville type theorems for a nonlinear elliptic equation. J. Math. 37, 977-986 (2017)

12. Wei, G.-F., Wylie, W.: Comparison geometry for the Bakry-Emery Ricci tensor. J. Differ. Geom. 83, 377-405 (2009)

\section{Submit your manuscript to a SpringerOpen ${ }^{\circ}$ journal and benefit from:}

- Convenient online submission

Rigorous peer review

- Open access: articles freely available online

High visibility within the field

- Retaining the copyright to your article 